\title{
Spectrum sensing methods for detection of DVB-T signals in AWGN and fading channels
}

\author{
Danyo Danev, Erik Axell and Erik G. Larsson \\ Department of Electrical Engineering (ISY), Linköping University, 58183 Linköping, Sweden
}

\begin{abstract}
In this paper, we consider spectrum sensing of DVB$T$ signals in different fading environments. We compare state-ofthe-art detectors including detectors based on pilot subcarriers, as well as detectors for general OFDM signals that exploit the correlation structure incurred by the cyclic prefix. Energy detection is also included for comparison. We show numerically that the choice of detector depends on the scenario, the detector requirements, and on the available prior knowledge. We also show that it is possible to obtain good detection performance by exploiting the correlation, even in a frequency selective channel.

Index Terms-spectrum sensing, DVB-T, OFDM, cognitive radio
\end{abstract}

\section{INTRODUCTION}

Recent measurements of spectrum utilization have shown unused spectrum resources in frequency, time and space [1], [2]. Cognitive radio is an emergent technique, intended to reuse these unused resources, or spectrum holes [3], [4]. The concept idea of cognitive radio is to adapt to the environment and use those spectrum resources that are available. However, introducing cognitive radios in an already existing primary network comes at a cost of increased interference. The impact on the primary network must be kept as small as possible. Therefore, secondary users must sense the spectrum and detect whether the primary system is using it or if there is a spectrum hole. In order to make this possible, the secondary users must be able to detect very weak primary user signals [5], [6].

The IEEE 802.22 working group is currently developing a cognitive radio standard, where spectrum sensing is included as a mandatory feature [7]. The IEEE 802.22 standard is intended for cognitive operation in the digital TV bands, among others. According to that standard, a secondary user must be able to detect a primary user DVB-T signal with probability of detection at least 0.9 and probability of false alarm no more than 0.1 , at $-22.2 \mathrm{~dB}$ SNR [8]. Cognitive radio operation in the DVB-T spectrum has also been considered in other research consortia (cf. [9]).

In this work, we evaluate numerous detection algorithms for detecting primary DVB-T signals, in different fading scenarios. We consider both a number of feature detectors, and as a comparison the energy detector. This is a natural expansion

The research leading to these results has received funding from the European Community's Seventh Framework Programme (FP7/2007-2013) under grant agreement no. 216076. This work was also supported in part by the Swedish Research Council (VR) and the Swedish Foundation for Strategic Research (SSF). E. Larsson is a Royal Swedish Academy of Sciences (KVA) Research Fellow supported by a grant from the Knut and Alice Wallenberg Foundation. of our work in [10] by incorporating the recently suggested methods of [11]. Another contribution is the investigation of the performance in the frequency selective extended vehicular A channel model defined in [12, B.2].

\section{Characteristics OF THE DVB-T Signal}

The ETSI document [13] specifies the physical layer characteristics of the DVB-T signal. The important parameters are summarized here and our main area of concern is the channel coding and modulation.

The digitalized multiplexed MPEG-2 signal which conveys the payload data is divided in packets of length 188 bytes. A Reed-Solomon (RS) [204, 188, 17] shortened code is used as an outer channel block code. This code is obtained by shortening of the original systematic RS [255,239,17] code with generator polynomial given in [13, Section 4.3.2]. The inner code constitutes of a rate $1 / 2$ convolutional code of constraint length 9 with 64 states. The generator polynomials $G_{1}=171_{\mathrm{OCT}}$ and $G_{2}=133_{\mathrm{OCT}}$ define this convolutional code. Predefined puncturing patterns are used in order to obtain the other available inner code rates, namely $2 / 3,3 / 4,5 / 6$ and $7 / 8$. The fading is mitigated by the introduction of interleaving.

The basic modulation constellations used are QPSK, 16QAM, 64-QAM, non-uniform 16-QAM or non-uniform 64QAM. Section 4.3.5 in [13] provides the specific mappings between the bit sequences and the constellation points. On top of that OFDM modulation is used.

The modulated DVB-T signal is determined by three main parameters, namely bandwidth, mode and length of the cyclic prefix. The signal bandwidth can take one of the values 5 $\mathrm{MHz}, 6 \mathrm{MHz}, 7 \mathrm{MHz}$ or $8 \mathrm{MHz}$. The number of subcarriers that are used in the OFDM modulation is determined by the mode parameter. This parameter can take the values $2 \mathrm{~K}$ or $8 \mathrm{~K}$ corresponding to 2048 and 8192 subcarriers, respectively. A cyclic prefix is commonly used in order to avoid the intersymbol interference (ISI) in wireless transmissions. The parameter determining the length of this cyclic prefix is defined in terms of fractions of the duration of the OFDM symbol part and admits the values $1 / 4,1 / 8,1 / 16$ and $1 / 32$.

A sequence of 68 consecutive OFDM symbols constitutes a frame and 4 such frames are gathered in a super frame. The continuous DVB-T signal in the time domain can be defined as

$$
s(t)=e^{j 2 \pi f_{c} t} \sum_{p=0}^{\infty} \sum_{l=0}^{67} \sum_{k=0}^{852 m} c_{p, l, k} \psi_{p, l, k}(t),
$$




\begin{tabular}{|c|c|c|c|c|c|c|}
\hline 0 & 48 & 54 & 87 & 141 & 156 & 192 \\
201 & 255 & 279 & 282 & 333 & 432 & 450 \\
483 & 525 & 531 & 618 & 636 & 714 & 759 \\
765 & 780 & 804 & 873 & 888 & 918 & 939 \\
942 & 969 & 984 & 1050 & 1101 & 1107 & 1110 \\
1137 & 1140 & 1146 & 1206 & 1269 & 1323 & 1377 \\
1491 & 1683 & 1704 & & & & \\
\hline
\end{tabular}

TABLE I

CONTINUAL PILOT CARRIER POSITIONS (INDEX K) FOR THE 2K MODE

with the basis functions $\varphi_{p, l, k}(t)$ determined as

$$
\varphi_{p, l, k}(t)=e^{j 2 \pi(k-426 m) 896 m\left[t-\Delta-l T_{S}-68 p T_{S}\right] /\left(10^{6} B\right)},
$$

whenever $(l+68 p) T_{S} \leq t<(l+68 p+1) T_{S}$ and 0 otherwise. Here $T_{S}$ is the OFDM symbol duration, $m \in\{2,8\}$ is the mode, $\Delta$ is the duration of the cyclic prefix and $f_{c}$ is the carrier frequency.

In order to ensure some guard interval between the DVB-T signals occupying neighboring channels some of the subcarriers are not utilized. For the $2 \mathrm{~K}$ and $8 \mathrm{~K}$ modes, the number of used carriers is 1705 and 6817 , respectively. The designated signal bandwidth $B$ (in $\mathrm{MHz}$ ) determines the elementary sampling period $T_{e}$ which is equal to $7 B / 64 \mu \mathrm{s}$. The subcarrier spacing is $10^{6} \mathrm{~B} /(896 \mathrm{~m}) \mathrm{Hz}$. This makes the total occupied bandwidth of the signal to be $213 B / 224 \mathrm{MHz}$ meaning that around $95.1 \%$ of the designated bandwidth is actually used.

There are three groups of utilized subcarriers. The modulated payload information is carried by the first and the largest group of subcarriers with a total number of $756 \mathrm{~m}$. The underlying QAM modulation have normalized (unit) mean energy. The second group (the TPS subcarriers) conveys information about the transmission parameters [13, Section 4.6]. The total number of subcarriers in this group is $8.5 \mathrm{~m}$ and their positions are fixed in each OFDM symbol. Finally, the third group represents the "boosted" pilots of mean energy $4 / 3$. The coefficient values depend on the subcarrier number and is calculated as

$$
c_{p, l, k}=\frac{4\left(1-2 w_{k}\right)}{3},
$$

where the sequence $\left\{w_{k}\right\}_{k=0}^{852 m}$ is obtained by a pseudo random binary sequence (PRBS) generator defined by the polynomial $X^{11}+X^{2}+1$ and initial state (11111111111). Table I gives the positions of the "boosted" pilots in the case of the $2 \mathrm{~K}$ mode. For the $8 \mathrm{~K}$ mode, these are given by $1704 i+k$, where $i=0,1,2,3$ and $k$ is a value in Table I. Additional "boosted" subcarriers are placed as $k$-th subcarrier in the $l$-th OFDM symbol of the frame whenever $3 \times(l \bmod 4)-k$ is divisible by 12 . Thus the total number of subcarriers in the third group is $1+87.5 m$, constituting approximately $10 \%$ of the used subcarriers.

\section{PRoblem Formulation}

Assume that the DVB-T signal mode is known, so that the parameters of the DVB-T signal that we want to detect is known. Let $x(t)$ be the received continuous baseband signal. Then, the time discrete received sequence is

$$
x[k]=x\left(k T_{e}\right), k=0,1, \ldots, M-1,
$$

where $M$ is the total number of samples.

The problem of spectrum sensing is to decide whether there is a signal transmitted or not. That is, we wish to discriminate between the following two hypotheses

$$
\begin{aligned}
& H_{0}: x[k]=n[k], k=0, \ldots, M-1, \\
& H_{1}: x[k]=\sum_{i=0}^{L-1} h_{i} s\left[k-k_{i}\right]+n[k], k=0, \ldots, M-1 .
\end{aligned}
$$

The hypotheses $H_{0}$ and $H_{1}$ correspond to absence and presence of a DVB-T signal, respectively. The sequence $s[k]$ is the sampled version of the signal $s(t)$ defined in (1), where $f_{c}=0$ and the sampling rate is $1 / T_{e}$. The model assumes that the signal propagates through multipath fading environment. The noise $n[i]$ is assumed to be complex white zero-mean Gaussian with variance $\sigma_{n}^{2}$.

The decision on the presence of a DVB-T signal is based on a test statistic $\Lambda$ which is a result of the received sequence $\{x[k]\}_{k=0}^{M-1}$, i.e

$$
\Lambda=f(x[0], x[1], \ldots, x[M-1]) .
$$

Various detection algorithms presented in the next section define this test statistic in a particular way. The decision upon the presence of the signal is based on the comparison of $\Lambda$ to a threshold $\eta$. The false alarm $\left(P_{f a}\right)$ and the probability of detection $\left(P_{d}\right)$ are given as

$$
P_{f a}=\operatorname{Pr}\left\{\Lambda>\eta \mid H_{0} \text { is true }\right\}
$$

and

$$
P_{d}=\operatorname{Pr}\left\{\Lambda>\eta \mid H_{1} \text { is true }\right\},
$$

respectively. The choice of the threshold is usually governed by the setting of $P_{f a}$ to a certain value.

\section{Detection Algorithms}

In this section we present the sensing algorithms which are considered for detection of the DVB-T signal.

\section{A. Energy Detection}

Energy detection, also known as radiometer [14], is probably the most widely used detector. The energy detector measures the received signal energy and compares it to a predefined threshold. That is, the test statistic for the energy detector is

$$
\Lambda_{\mathrm{ED}} \triangleq \sum_{k=0}^{M-1} x[k] x^{*}[k]
$$

The performance of the energy detector is well known (cf. [15]) and can be written in closed form. The probability of false alarm $P_{\mathrm{FA}}$ is given by

$$
P_{\mathrm{FA}} \triangleq \operatorname{Pr}\left(\Lambda_{\mathrm{ED}}>\eta_{\mathrm{ED}} \mid H_{0}\right)=1-F_{\chi_{2 M}^{2}}\left(\frac{2 \eta_{\mathrm{ED}}}{\sigma_{n}^{2}}\right),
$$


where $F_{\chi_{2 M}^{2}}(\cdot)$ denotes the cumulative distribution function of a $\chi^{2}$-distributed random variable with $2 M$ degrees of freedom. Thus, given a false alarm probability, we can derive the threshold $\eta_{\mathrm{ED}}$ from

$$
\eta_{\mathrm{ED}}=F_{\chi_{2 M}^{2}}^{-1}\left(1-P_{\mathrm{FA}}\right) \frac{\sigma_{n}^{2}}{2} .
$$

The probability of detection is then given by

$$
P_{\mathrm{D}} \triangleq \operatorname{Pr}\left(\Lambda_{\mathrm{ED}}>\eta_{\mathrm{ED}} \mid H_{1}\right)=1-F_{\chi_{2 M}^{2}}\left(\frac{2 \eta_{\mathrm{ED}}}{\sigma_{n}^{2}+\sigma_{s}^{2}}\right),
$$

where $\sigma_{s}^{2}$ is the average received signal power. From 5 it is clear that the energy detector requires the noise power $\sigma_{n}^{2}$ to be known. Moreover, it is well known that the performance of the energy detector quickly deteriorates when the noise power estimate is imperfect [6], [16].

\section{B. Detection Based on Second-Order Statistics}

Much literature consider spectrum sensing of OFDM signals. The repetition of data in the cyclic prefix $(\mathrm{CP})$ incurs a certain correlation structure to an OFDM signal. This structure can be exploited for detection. In the following sections, we will briefly describe a few state-of-the-art feature detectors for OFDM signals. First, we will describe the detectors of [11], which are based on second-order statistics of the received signal. Let $T_{d}=T_{S} / T_{e}$ be the number of data samples in an OFDM symbol, and $T_{c}$ be the number of samples in the CP. As a preliminary, define the sample value product

$$
r[k] \triangleq x[k]^{*} x\left[k+T_{d}\right], k=0, \ldots, M-T_{d}-1
$$

Because of the repetition of data in the CP, $x[k]$ and $x\left[k+T_{d}\right]$ are correlated and $E[r[k]] \neq 0$ for those samples belonging to the CP. Moreover, $E[r[k]]=0$ for samples not in the CP. Now, if $x[k]$ and $x\left[k+T_{d}\right]$ are correlated in the first OFDM symbol, the corresponding samples $x\left[k+i\left(T_{c}+T_{d}\right)\right]$ and $x\left[k+T_{d}+\right.$ $\left.i\left(T_{c}+T_{d}\right)\right]$ in all other symbols are also correlated. That is, if $E[r[k]] \neq 0$, then $E\left[r\left[k+i\left(T_{c}+T_{d}\right)\right]\right] \neq 0$. Analogously, if $E[r[k]]=0$, then $E\left[r\left[k+i\left(T_{c}+T_{d}\right)\right]\right]=0$. Thus, define the average sample value product

$R[k] \triangleq\left\{\begin{array}{l}\frac{1}{K+1} \sum_{i=0}^{K-1} r\left[k+i\left(T_{c}+T_{d}\right)\right], k=0, \ldots, n_{K}-1, \\ \frac{1}{K} \sum_{i=0}^{K-2} r\left[k+i\left(T_{c}+T_{d}\right)\right], k=n_{K}, \ldots, T_{c}+T_{d}-1,\end{array}\right.$

where $K \triangleq\left\lfloor\frac{M-T_{d}}{T_{c}+T_{d}}\right\rfloor$, and $n_{K}=M-T_{d} \quad \bmod \left(T_{c}+T_{d}\right)$. That is, $K$ is the number of OFDM symbols over which all sample value products can be averaged, and $n_{K}$ is the number of sample value products that can be averaged over $K+1$ symbols. That is, if the number of samples $M=K\left(T_{c}+\right.$ $\left.T_{d}\right)+T_{d}$, all sample value products will be averaged over $K$ OFDM symbols.

Then, the test statistic of [11] is

$$
\max _{\theta} \frac{\sum_{i=0}^{T_{c}+T_{d}-1}|R[i]|^{2}}{\sum_{k \in S_{\theta}}\left|R[k]-\frac{1}{T_{c}} \sum_{i \in S_{\theta}} \operatorname{Re}(R[i])\right|^{2}+\sum_{j \notin S_{\theta}}|R[j]|^{2}},
$$

where $S_{\theta}=\left\{\theta, \ldots, \theta+T_{c}-1\right\}^{T} \bmod \left(T_{c}+T_{d}\right)$ are the indices of the $T_{c}$ "consecutive" correlated samples given $\theta$. This detector is assumed to know the size of the OFDM symbols, that is $T_{c}$ and $T_{d}$, and exploits the structure incurred by the cyclic prefix. Note however, that this detector does not require any knowledge of the noise power, in contrary to the energy detector.

Another detector was proposed in [11], if the noise power is known (or estimated). Then the test statistic proposed in [11] becomes (when all constants are removed)

$$
\max _{\theta}\left(\sum_{k \in S_{\theta}} \operatorname{Re}(R[k])\right)^{2}
$$

\section{Autocorrelation-Based Detection}

In this section we will describe the detector of [17], which also exploits the correlation of an OFDM signal, using knowledge of $T_{d}$. The method of [17] was called an autocorrelationbased detector and it uses the empirical mean of the sample value products $r[k]$, normalized by the received power, as test statistic. More precisely, the test proposed in [17] is

$$
\frac{\frac{1}{M-T_{d}} \sum_{k=0}^{M-T_{d}-1} \operatorname{Re}(r[k])}{\frac{1}{M} \sum_{k=0}^{M-1}|x[k]|^{2}} \text {. }
$$

Just like (9), the detector (11) does not require any knowledge about the noise power $\sigma_{n}^{2}$.

\section{CP-Based Sliding Window}

The detector of [18] uses a sliding window that sums over $T_{c}$ consecutive samples, and takes the maximum. The test statistic is

$$
\max _{\theta}\left|\sum_{k=\theta}^{\theta+T_{c}-1} r[k]\right| .
$$

The statistic (12) only takes one OFDM symbol at a time into account. One way of extending this detector for multiple symbols and an arbitrary number of samples, is to use the test statistic

$$
\max _{\theta}\left|\sum_{k=\theta}^{\theta+T_{c}-1} R[k]\right|
$$

Similar to this we could alternatively take the real part of the sum instead of the absolute value as in (13). This approach was proposed in [10], and gives the test statistic

$$
\max _{\theta} \operatorname{Re}\left(\sum_{k=\theta}^{\theta+T_{c}-1} R[k]\right) \text {. }
$$

Note the similarity of the detectors (13)-(14) and the detector (10) based on second-order statistics. Just like the detector (10), the detectors (13) and (14) has the drawback that $\sigma_{n}^{2}$ needs to be known.

It has been shown in simulations that the detector taking the real part (14) performs better than the detector taking the absolute value (13) [10]. Therefore, we will not include the detector (13) in our numerical results. 


\section{E. Detection Based on the IFFT of Pilot}

The DVB-T signal contains pilot subcarriers, used for estimation of the channel. The detector of [10] exploits these pilot subcarriers for detection. Due to the fact that the coefficients $c_{p, k, l}$ are fixed and known, the performance of this detector is superior to the others presented here. However, the knowledge of the parameters of the DVB-T signal are essential for this algorithm to work properly.

In order to describe the test statistic we first define the function

$$
s_{p}(t)=e^{j 2 \pi f_{c} t} \sum_{p, l, k:(l, k) \in A} c_{p, l, k} \psi_{p, l, k}(t),
$$

where the set $A$ defines the positions of the pilots in the third group of utilized carriers (see Section II). This deterministic signal $s_{p}(t)$ is sampled at the rate $1 / T_{e}$ to obtain the sequence

$$
s_{p}[n]=s_{p}\left(n T_{e}\right), \quad n=0,1, \ldots
$$

This sequence is cyclic with the period $4\left(T_{c}+T_{d}\right)$, which means that it can be completely determined by the vector $\left(s_{p}[0], s_{p}[1], \ldots, s_{p}\left[4\left(T_{c}+T_{d}\right)-1\right]\right)$.

The test statistic of the pilot based detector is now given as

$$
\max _{\theta \in\left\{0,1, \ldots, 4\left(T_{d}+T_{c}\right)-1\right\}}\left|\sum_{k=0}^{M-\theta-1} s_{p}[k] x^{*}[k+\theta]\right| .
$$

The determination of the threshold value has to be performed empirically. This is usually done by feeding the detector with pure noise signals and calculating the test statistic. The estimate of the threshold is obtained by choosing the level that gives the desired $P_{f a}$. Usually, around 1000 simulations are enough to get a reasonable estimate on the threshold.

\section{Numerical Results}

Here we present comparisons of the described spectrum sensing algorithms for some particular scenarios. The compared detectors are summarized in Table II. The transmission of a DVB-T signal of bandwidth $8 \mathrm{MHz}$ with underlying non-uniform 16-QAM modulation is assumed. We consider propagation of the signal in Rayleigh or Rice fading channels, using the built-in MatLab fading generators.

We consider six different scenarios, with different channel models and sensing time. The DVB-T signal model is the same for all scenarios. The following settings are common for all scenarios:

- DVB-T signal mode: $2 \mathrm{~K}$;

- cyclic prefix: $T_{c} / T_{d}=1 / 4$

- false alarm probability: $P_{f a}=0.05$;

The compared detectors are summarized in Table II

\section{Scenario 1: AWGN (Figure 1)}

In the first scenario, we consider an AWGN channel with $10 \mathrm{~ms}$ sensing time. The results are shown in Figure 1.

Scenario 2: Rayleigh flat fading (Figure 2)

In this scenario, we consider a Rayleigh flat fading channel with $10 \mathrm{~ms}$ sensing time. The results are shown in Figure 2.

Scenario 3: Rayleigh flat fading (Figure 3)

\begin{tabular}{|l|l|l|l|}
\hline Detector & Ref. & Test & Prior knowledge \\
\hline (i) Pilot & {$[10]$} & $(16)$ & $\sigma_{n}^{2}, T_{d}, T_{c}, c_{p, l, k}, A$ \\
\hline (ii) Energy & {$[14]$} & $(3)$ & $\sigma_{n}^{2}$ \\
\hline (iii) Sliding Window (SW) & {$[18]$} & $(14)$ & $\sigma_{n}^{2}, T_{d}, T_{c}$ \\
\hline (iv) 2nd-order (known $\sigma_{n}^{2}$ ) & {$[11]$} & $(10)$ & $\sigma_{n}^{2}, T_{d}, T_{c}$ \\
\hline (v) 2nd-order & {$[11]$} & $(9)$ & $T_{d}, T_{c}$ \\
\hline (vi) Autocorrelation (AC) & {$[17]$} & $(11)$ & $T_{d}$ \\
\hline
\end{tabular}

TABLE II

SUMMARY OF DETECTORS.

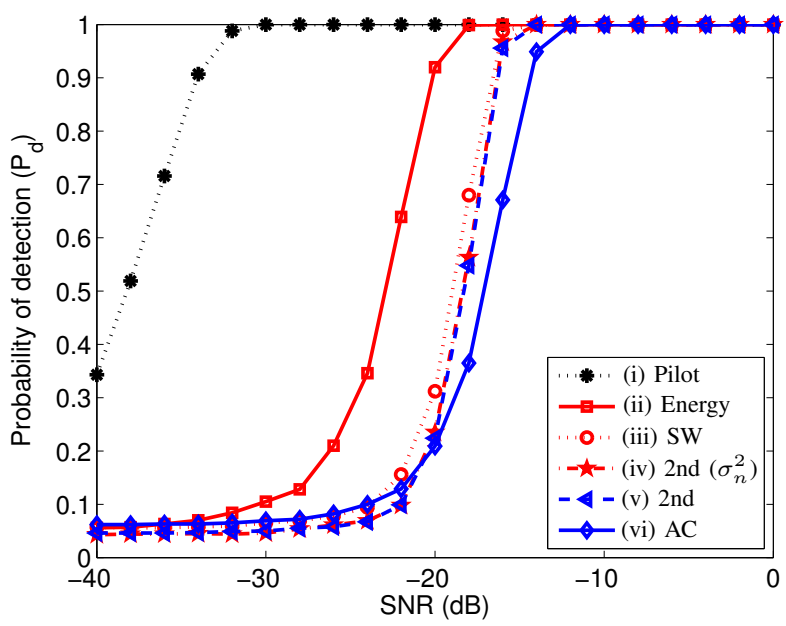

Fig. 1. Probability of detection as a function of SNR for AWGN channel, with $10 \mathrm{~ms}$ sensing time. The detectors are summarized in Table II.

In the third scenario, we consider a Rayleigh flat fading channel with $50 \mathrm{~ms}$ sensing time. That is, the sensing time is increased compared to Scenario 2. The results are shown in Figure 3.

\section{Scenario 4: Rice fading (Figure 4)}

In the fourth scenario, we consider a Rice fading channel $(\mathrm{K}=5)$ with $10 \mathrm{~ms}$ sensing time. The results are shown in Figure 4.

Scenario 5: Rayleigh flat fading and shadowing (Figure 5)

In this scenario, we consider a Rayleigh fading channel with shadowing, and $10 \mathrm{~ms}$ sensing time. The standard deviation of the log-normal shadowing is $6 d B$. The results are shown in Figure 5.

\section{Scenario 6: Multipath Rayleigh fading (Figure 6)}

In this last scenario, we consider a multipath Rayleigh fading channel as described by the extended vehicular A channel model given in [12, B.2]. The sensing time is again $10 \mathrm{~ms}$. The results are shown in Figure 6.

The SNR is calculated as the ratio between the mean received power of the signal and the noise variance. The simulated channel models are realized by the build-in functions in MATLAB ${ }^{\circledR}$ for the Rayleigh and Rician fading.

The graphs show that utilizing the features of the DVB-T signal can provide reliable detection at rather low SNRs in the case of frequency flat fading. However, this requires a priori 


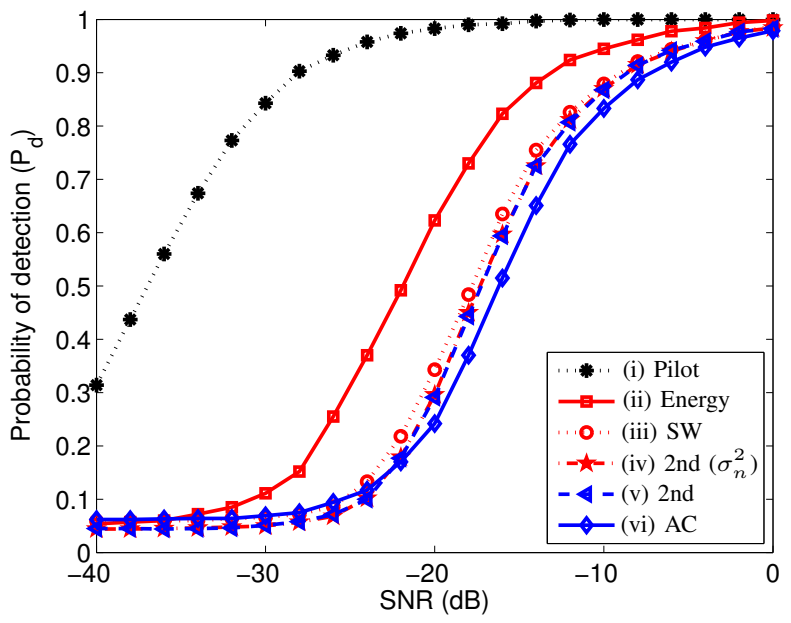

Fig. 2. Probability of detection as a function of SNR for Rayleigh flat fading, with $10 \mathrm{~ms}$ sensing time. The detectors are summarized in Table II.

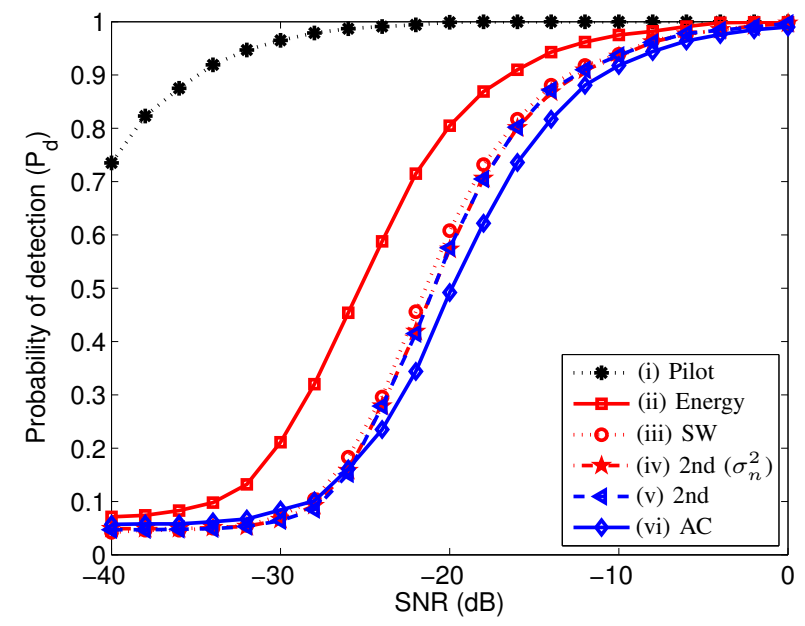

Fig. 3. Probability of detection as a function of SNR for Rayleigh flat fading with $50 \mathrm{~ms}$ sensing time. The detectors are summarized in Table II.

knowledge of the parameters for the transmitted signal. For the frequency selective channel of Scenario 6, we see that the performance of the pilot based detector deteriorates. This can be explained by the fact that the frequency selective fading influences the pilots differently. In order for this detector to work well, a good estimation of the channel is needed. The performance can then be improved by properly adjusting the time domain signal generated by the pilots.

Apart from the pilot based detector, the other presented algorithms do not rely on the information about the structure of the waveform. The only assumption is that the length of the cyclic prefix and the total duration of the OFDM symbol are known for the feature detectors (iii)-(vi), while the energy detector (ii) does not require any knowledge about the signal. The energy detector tends to show the best performance among these, but its drawback is that it assumes perfect knowledge

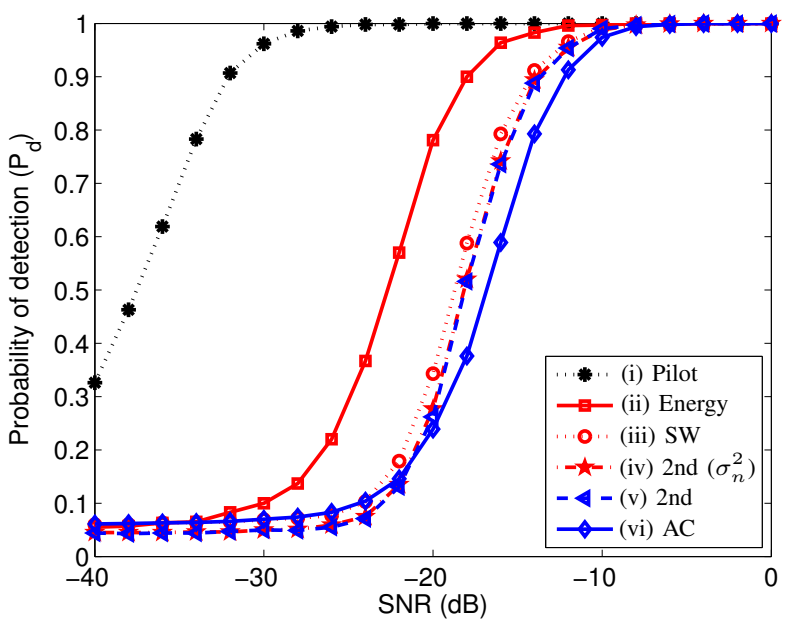

Fig. 4. Probability of detection as a function of SNR for Rice fading $(K=5)$, with $10 \mathrm{~ms}$ sensing time. The detectors are summarized in Table II.

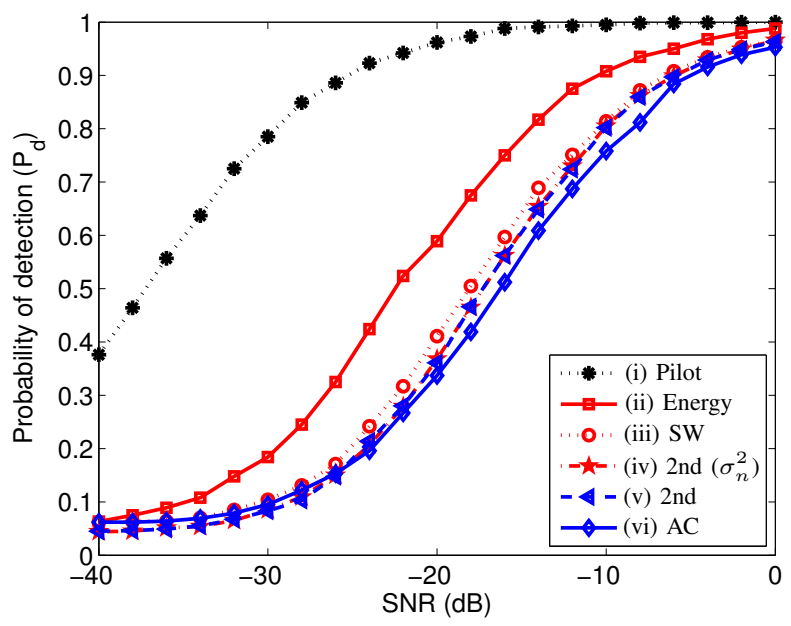

Fig. 5. Probability of detection as a function of SNR for Rayleigh flat fading and shadowing, with $10 \mathrm{~ms}$ sensing time. The detectors are summarized in Table II.

of the noise floor level. Thus, if the noise power is known, the energy detector works quite well. Then, there is no gain in exploiting the correlation of the OFDM signal as done by the sliding window detector (iii) and the second-order detector (iv). Similar conclusions were made in [11] for an AWGN channel. As already mentioned, the performance of the energy detector is severely decreased if the noise power knowledge is erroneous. In practical scenarios, the noise power is estimated, and never perfectly known. Moreover, if there is interference in addition to the noise, for example from another secondary user, the feature detectors would be able to distinguish the primary signal from the interfering signal, whereas the energy detector would not.

The two second-order methods (iv)-(v) of Section IV-B perform almost equally in all scenarios. Thus, when using 


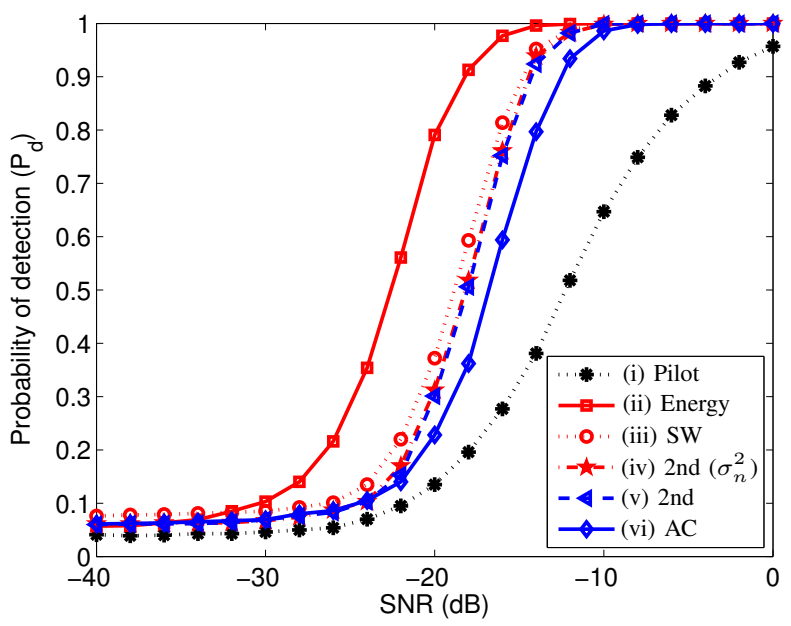

Fig. 6. Probability of detection as a function of SNR for multipath fading, with $10 \mathrm{~ms}$ sensing time. The detectors are summarized in Table II.

the second-order method, one can only expect a very small gain from knowing the noise power for sensing times above $10 \mathrm{~ms}$ as shown here. This shows that knowledge of the noise floor level is in practise not necessary in this case. If the noise power is completely unknown, only the second-order statistics detector (v) of [11] and the autocorrelation detector (vi) of [17] work. A general observation is that the second-order method (v) slightly outperforms the autocorrelation-based detector (vi) in all scenarios, in the region $P_{d} \gtrsim 0.15$. We argue that a probability of detection below this level will not be acceptable in a real situation, as it would cause to much interference to the primary users. In most of the scenarios the performance gain at the probability of detection of $95 \%$ is at least $1 d B$.

Another observation is that the frequency selectivity of the channel does not influence much the performance of the presented algorithms, with the exception of the pilot based detector. Thus, even when the channel is frequency selective, the correlation of an OFDM signal incurred by the cyclic prefix can be exploited for detection.

\section{CONCLUDING REMARKS}

In this paper we have considered various sensing techniques for detection of DVB-T signals. In future cognitive usage of the frequency spectrum occupied by digital terrestrial television broadcasts, the reliable detection of the presence of a transmission will be essential. The second-order statistics methods seem to provide good performance with less information and are good candidates for implementation in cognitive radio spectrum sensors.

In this work, we did not include carrier frequency offset. Such source of imperfect knowledge will decrease the correlation structure of the OFDM signal. Thus, the performance of the feature detectors would decrease. Comparisons of spectrum sensing algorithms under such circumstances should be considered in future work.

\section{REFERENCES}

[1] FCC, "Spectrum policy task force report," Tech. Rep. 02-135, Federal Communications Commission, November 2002, Available: http://hraunfoss.fcc.gov/edocs_public/attachmatch/DOC-228542A1.pdf.

[2] M.A. McHenry, "NSF spectrum occupancy measurements project summary," Tech. Rep., SSC, August 2005, Available: http://www.sharedspectrum.com/.

[3] S. Haykin, "Cognitive radio: brain-empowered wireless communications," IEEE Journal on Selected Areas in Communications, vol. 23, no. 2, pp. 201-220, Feb. 2005.

[4] I. J. Mitola, "Software radios: Survey, critical evaluation and future directions," IEEE Aerospace and Electronic Systems Magazine, vol. 8, no. 4, pp. 25-36, Apr 1993.

[5] E. Larsson and M. Skoglund, "Cognitive radio in a frequency-planned environment: some basic limits," IEEE Transactions on Wireles Communications, vol. 7, no. 12, pp. 4800-4806, December 2008.

[6] A. Sahai, N. Hoven, and R. Tandra, "Some fundamental limits on cognitive radio," in Allerton Conference on Communication, Control, and Computing, October 2004, pp. 1662-1671.

[7] C. Stevenson, G. Chouinard, Zhongding Lei, Wendong Hu, S. Shellhammer, and W. Caldwell, "Ieee 802.22: The first cognitive radio wireless regional area network standard," IEEE Communications Magazine, vol. 47, no. 1, pp. $130-138$, january 2009.

[8] S. Shellhammer and G. Chouinard, "Spectrum sensing requirements summary," IEEE Std.802.22-06/0089r4, June 2006.

[9] SEnsor Network for Dynamic and cOgnitive Radio Access (SENDORA), , http://www.sendora.eu.

[10] D. Danev, "On signal detection techniques for the DVB-T standard," in Proceedings of the 4th International Symposium on Communications, Control and Signal Processing, March 2010.

[11] E. Axell and E. G. Larsson, "Optimal and near-optimal spectrum sensing of OFDM signals in AWGN channels," Workshop on Cognitive Information Processing (CIP), 2010, Accepted.

[12] 3rd Generation Partnership Project, "3GPP TS 36.104 V8.9.0," Tech. Rep. 36.104, March 2010, Available: http://www.3gpp.org/ftp/Specs/latest/Rel-8/36_series/36104-890.zip.

[13] ETSI EN 300744 V1.6.1 (2009-01), "Digital video broadcasting (DVB); framing structure, channel coding and modulation for digital terrestrial television,” Tech. Rep., ETSI, 2009.

[14] H. Urkowitz, "Energy detection of unknown deterministic signals," Proceedings of the IEEE, vol. 55, no. 4, pp. 523-531, April 1967.

[15] H. L. Van Trees, Detection, Estimation, and Modulation Theory: Part I, John Wiley and Sons, Inc., 1968.

[16] R. Tandra and A. Sahai, "Fundamental limits on detection in low SNR under noise uncertainty," in IEEE International Conference on Wireless Networks, Communications and Mobile Computing, June 13-16 2005, vol. 1 , pp. 464-469.

[17] S. Chaudhari, V. Koivunen, and H. V. Poor, "Autocorrelation-based decentralized sequential detection of OFDM signals in cognitive radios," IEEE Transactions on Signal Processing, vol. 57, no. 7, pp. 2690-2700, July 2009.

[18] Huawei Technologies and UESTC, "Sensing scheme for DVB-T," IEEE Std.802.22-06/0127r1, July 2006. 